Swarthmore College

Works

6-17-1996

\title{
Experimental Evidence For Self-Similar Structures In The Aggregation Of Porphyrins In Aqueous Solutions
}

\author{
F. Mallamace
}

N. Micali

S. Trusso

L. M. Scolaro

A. Romeo

See next page for additional authors

Follow this and additional works at: https://works.swarthmore.edu/fac-chemistry

Part of the Inorganic Chemistry Commons

Let us know how access to these works benefits you

\section{Recommended Citation}

F. Mallamace, N. Micali, S. Trusso, L. M. Scolaro, A. Romeo, A. Terracina, and Robert F. Pasternack. (1996). "Experimental Evidence For Self-Similar Structures In The Aggregation Of Porphyrins In Aqueous Solutions". Physical Review Letters. Volume 76, Issue 25. 4741-4744. DOI: 10.1103/PhysRevLett.76.4741 https://works.swarthmore.edu/fac-chemistry/118

This work is brought to you for free by Swarthmore College Libraries' Works. It has been accepted for inclusion in Chemistry \& Biochemistry Faculty Works by an authorized administrator of Works. For more information, please contact myworks@swarthmore.edu. 


\section{Authors}

F. Mallamace, N. Micali, S. Trusso, L. M. Scolaro, A. Romeo, A. Terracina, and Robert F. Pasternack 


\title{
Experimental Evidence for Self-Similar Structures in the Aggregation of Porphyrins in Aqueous Solutions
}

\author{
F. Mallamace \\ Dipartimento di Fisica, Universita' di Messina, Vill. S. Agata, P.O. Box 55, I-98166, Messina, Italy \\ N. Micali and S. Trusso \\ Istituto di Tecniche Spettroscopiche del CNR, C. da Papardo, Salita Sperone, 31, I-98166, Messina, Italy \\ L. Monsu' Scolaro, A. Romeo, and A. Terracina \\ Dipartimento di Chimica Inorganica, Chimica Analitica e Chimica Fisica dell' Universita' di Messina and ICTPN-CNR, \\ Sezione di Messina, Messina, Italy \\ R. F. Pasternack \\ Department of Chemistry, Swarthmore College, Swarthmore, Pennsylvania 19081
}

(Received 15 February 1996)

\begin{abstract}
We have studied the aggregation of the porphyrin $t-\mathrm{H}_{2} \mathrm{P}_{\mathrm{agg}}$ in aqueous solution by light scattering. The intensity profile of the elastically scattered light, studied in the exchanged wave-vector range $0.2 \leq q \leq 31.4 \mu \mathrm{m}^{-1}$, indicates that the aggregation produces large monodisperse clusters having a fractal structure, and is driven by diffusion-limited aggregation kinetics. Additional measurements performed at different $q$ values confirm such a picture giving a hydrodynamic radius $R_{H}$ consistent with the radius of gyration $R_{g}$ measured by elastic scattering. This is explained taking into account the $q^{2}$ dependence observed in the mean decay rate of the intensity-intensity correlation function and the effect on this latter dynamical quantity of anisotropies in the cluster structure. [S0031-9007(96)00430-9]
\end{abstract}

PACS numbers: 64.60.Cn, 05.40.+j, 82.70.Dd

Aggregation phenomena are of central interest in many fields of science and technology, and in particular in research areas that are at the borderline between physics, chemistry, and biology. Monomer aggregation, in the buildup of large supramolecular assemblies, is relevant to the understanding of systems such as polymers, gels, colloids, biological macromolecules, and various nucleation processes involved at phase transitions [1]. The concept of fractal geometry and the application of the scaling laws have proven to be successful for the description of nonequilibrium aggregation in many of these systems [2]. A large variety of techniques, such as electron microscopy, x-ray and light scattering, as well as computer simulations, make apparent the signature of fractal structures originating from different kinetics of growth. In fractal aggregates, the various growth mechanisms relate the cluster mass $M$ to the corresponding radius $R$ via the well known scaling form $M \propto R^{d_{f}}$ [3], where the fractal or Hausdorff dimension $d_{f}$ differs from the spatial (Euclidean) dimension $d$. The different growth processes [i.e., percolation, simple diffusion-limited aggregation (DLA), reaction-limited cluster-cluster aggregation (RLA), diffusion-limited cluster-cluster aggregation (DLCCA), etc.] are characterized by different values in the corresponding $d_{f}$. Therefore this quantity can be considered as an indicator of the nature of the aggregation kinetics [2]. For example, charged colloidal particles (latex spherical particles) can be caused to aggregate, upon the addition of salt, with a fractal dimension $d_{f} \cong 2.1$, a value which suggests a RLA mechanism. Other systems show alternative kinetic processes of growth exhibiting $\operatorname{DLCCA}\left(d_{f} \cong 1.75\right)$ or DLA $\left(d_{f} \cong 2.5\right)[1,2]$.

Although initially scattering techniques were used solely to probe geometrical aspects of aggregates, they now constitute a powerful experimental tool to explore the dynamical properties of these structures. This approach yields detailed relationships between their hydrodynamic and static properties. It is the purpose of this Letter to address the fractal properties of aqueous porphyrin solutions by means of both elastic and quasielastic light scattering experiments.

It is well known that porphyrins constitute an important class of chemical compounds. A relatively recent and interesting application of synthetic cationic porphyrins (and their metalloderivatives) in biophysics is represented by their use as probes of the dynamics and the structure of nucleic acids and helical polypeptides [4]. In particular, these species can bind to DNA and RNA by intercalation, external groove binding, and external binding with self-stacking. The observed interaction is dependent on the porphyrin structure, the ionic strength, and the polynucleotide composition [4-6]. The rapid uptake of these molecules by DNA supports the idea of a "collective motion" model for nucleic acids, involving the simultaneous breaking of multiple adjacent hydrogen bonds [4]. Porphyrins can induce conformational changes into the double helix, for example, converting Z-DNA to the B-form [4]. The use of an electron 
acceptor porphyrin is a powerful tool for the investigation of energy transfer processes through the double helix of DNA [4] and several metalloderivatives have been reported as showing nuclease activity, making these compounds useful reagents for footprinting experiments [4]. A particular behavior is shown by aqueous solutions of the dicationic porphyrin trans-bis $(N$-methylpyridinium-4yl)diphenylporphyine $\left(t-\mathrm{H}_{2} \mathrm{P}_{\mathrm{agg}}\right)$. Upon addition of salt, this porphyrin forms supramolecular assemblies even in the absence of the biopolymer [5,6]. This self-aggregation has been revealed by the results of different experimental techniques such as UV-visible, resonance Rayleigh light scattering (RRLS), and, in the presence of helical templates, circular dichroism (CD) spectroscopy [5,6]. Although these techniques have given information on the presence of large structures of porphyrin molecules, they do not give details on the nature of these clusters.

The samples were prepared at $298 \mathrm{~K}$ by using the free base porphyrin $t-\mathrm{H}_{2} \mathrm{P}_{\mathrm{agg}}$ as the chloride salt. The studied concentration, $5 \times 10^{-6} \mathrm{M}$, was determined by using $\varepsilon=2.40 \times 10^{5} \mathrm{M}^{-1} \mathrm{~cm}^{-1}$ at the Soret maximum [6], at zero ionic strength. Aggregation was induced by adding $\mathrm{NaCl}$ up to a final concentration of $0.1 \mathrm{M}$. Elastic light scattering measurements were performed in two ranges of scattering angle $\theta$ by using two different experimental systems. In the range $20^{\circ} \leq \theta \leq 150^{\circ}$, corresponding to scattering wave vectors $5.6 \leq q \leq 31.4 \mu \mathrm{m}^{-1}$, a computerized goniometer was used (ELS); whereas the small angle region (SALS) in the wave-vector range $0.21 \leq$ $q \leq 1.99 \mu \mathrm{m}^{-1}$ was explored by using a homemade apparatus. Dynamic light scattering (DLS) was studied in the same angular range as ELS by using a Malvern 4700 submicron particle analyzer system. With this apparatus the intensity-intensity correlation function was measured in the time range $10 \mu \mathrm{s} \leq \tau \leq 1 \mathrm{~s}$. The light source for ELS and DLS was the $514.5 \mathrm{~nm}$ line of an argon laser operating at a power of $200 \mathrm{~mW}$. The experimental setup for SALS has been described in a previous paper [7]. This optical apparatus is based on performing an optical Fourier transform of the impinging (scattered) wave front at a linear position sensitive detector. In the present case the exciting light source is a $10 \mathrm{~mW}$ $\mathrm{HeNe}$ laser $(632.8 \mathrm{~nm})$. A 1024 element diode array was used as detector and a 16 bit analog-to-digital converter was employed to digitalize the signal. The intensity scattered by the optical cell filled by the solvent was taken as the background and subtracted from all the measured spectra. Furthermore, data coming from ELS were scaled by a multiplicative factor in order to perform the fitting procedure.

The method for obtaining the aggregate fractal dimension involves the measurement of the scattered intensity as a function of the exchanged wave vector. The measured intensity is $I(q) \propto P(q) S(q)$ [8], where $P(q)$ is the form factor of the monomeric unit and $S(q)$ is the structure factor [i.e., the Fourier transform of the density correlation function $g(r)]$. In the actual experimental situation, for the $q$ range used and given that the porphyrin molecule is the monomeric unit, $P(q) \simeq 1$ [8]. In addition, if $S_{M}(q)$ represents the structure factor of a cluster of mass $M$ and size $R\left[M=M_{0} k\right.$ ( $M_{0}$ being the mass of a monomer and $k$ the number of monomers with a size $\left.R_{0}\right)$ ], it is shown that for a fractal structure, $S_{M}(q) \propto S(q R)$ [9]. Under the conditions $q R_{0} \ll 1$ and $q R \gg 1$,

$$
I(q) \propto S(q) \propto q^{-d_{f}} .
$$

If the intensity $I(q)$ is plotted on a double logarithmic scale as a function of $q$, a straight line with slope $-d_{f}$ is obtained. However, such a condition is not fulfilled even when the size of the scatterers grows up to the micrometer scale. In such a case, the obtained scattering profile must be compared to a $I(q)$ form calculated for a fractal system $[10,11]$. For fractal objects, the density correlation function has the characteristic of being homogeneous:

$$
g(r)=\frac{1}{\rho} \frac{d_{f}}{4 \pi}\left(R_{0}^{d_{f}}\right)^{-1} r^{d_{f}-3} \exp \left(-\frac{r}{\xi}\right),
$$

where $\xi$ is a cutoff parameter (equal to the radius of the cluster); Chen and Texeira [10] were able to deduce the structural factor for a fractal aggregate of $k$ monomers. Their result can be written as

$$
S_{k}(q)=\frac{k \sin \left[\left(d_{f}-1\right) \arctan \left(q R_{k}\right)\right]}{\left(d_{f}-1\right) q R_{k}\left(1+q^{2} R_{k}^{2}\right)^{\left(d_{f}-1\right) / 2}},
$$

where $R_{k}=\xi$ is the cluster radius and is related to $R_{0}$ by $R_{k}=R_{0} k^{-d_{f}}$ [2,3]. By considering the cluster polydispersity, and a proper cluster size distribution function $N(k)$, the total scattered intensity $I(q)$ can be obtained as

$$
I(q)=\int_{1}^{\infty} k N(k) S_{k}(q) d k .
$$

In Fig. 1 the scattered intensity profiles obtained by SALS and ELS are reported. The continuous line is the best fit by Eq. (3) with the multiplicative factor $A$, as well as $\xi$ and $d_{f}$ as free parameters. As can be observed, data coming from two different types of measurements (ELS and SALS) fall on the same curve. The good agreement with Eq. (3) implies that the system aggregation gives rise to nearly monodisperse clusters. From the data fitting we obtain $d_{f} \cong 2.5$ and $\xi \cong 3.0 \mu \mathrm{m}$. This $\xi$ value indicates the presence in the solution of very large aggregates. The value obtained for $d_{f}$ together with the additional result that clusters are nearly monodisperse suggests that (inside the experimental uncertainty) the aggregation kinetics are of the diffusion limited DLA type [2].

The growth of large aggregates in the porphyrin solutions under investigation can be confirmed by means of quasielastic light scattering data (DLS). In addition, by considering the self-similar structure of the diffusing objects, it can be shown that there is good agreement 


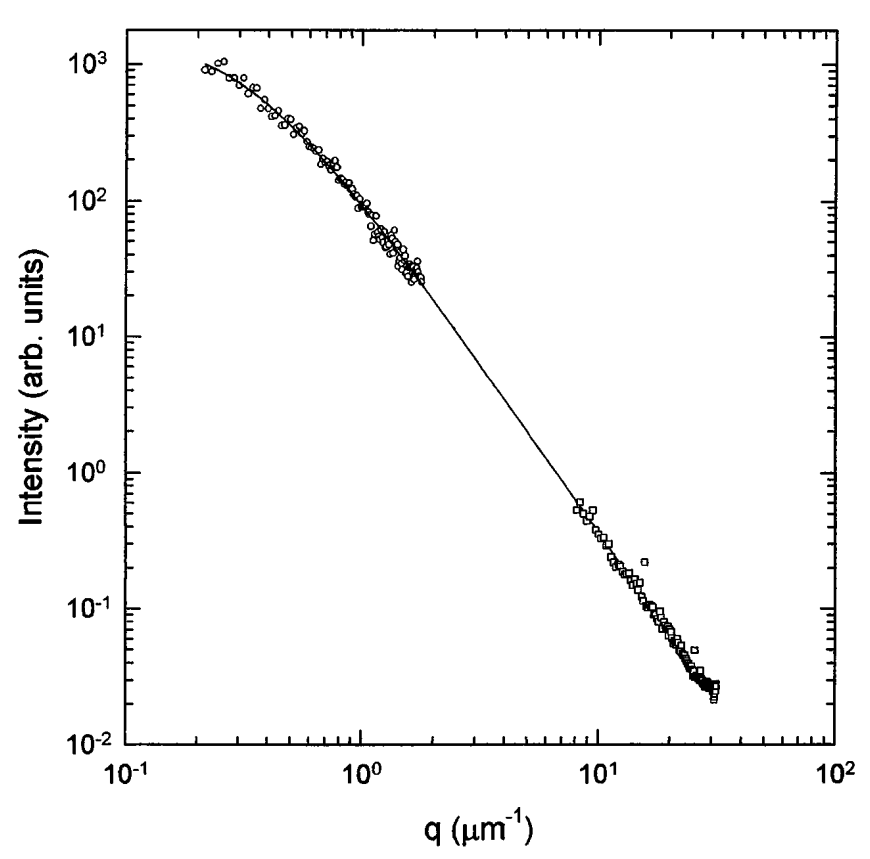

FIG. 1. Intensity profile $I(q)$ vs $q$ in the regime of complete aggregation. Open circles are the experimental data coming from SALS and squares from ELS, continuous line is the best fit with Eq. (3).

between the cluster size measured from the static (combination of SALS an ELS data) and dynamical data (DLS).

The same scaling form used for the structure factor $S(q)$ and the theoretical models of the kinetic growth for fractal aggregates can be used to obtain information on the dynamical properties of these self-similar structures $[12,13]$. In a DLS homodyne experiment, the dynamic structure factor $S(q, \tau)$ is measured as proportional to the autocorrelation function of the scattered field. From this quantity the mean decay rate (weighted by the cluster size distribution) $\Gamma_{c}$ is obtained as the first cumulant:

$$
\Gamma_{c}(q)=\frac{q^{2}}{I(q)} \int_{1}^{\infty} k N(k) S_{k}(q)\left[D q^{2}+A\right] d k,
$$

where $D$ and $A$ are the translational and rotational diffusion coefficients of a cluster of mass $M$, respectively. Depending on the radius of gyration $R_{g}$ of a cluster, one of two distinct dependence of $\Gamma_{c}$ on $q$ can result. When $q R_{g} \ll 1, \Gamma_{c} \propto q^{2}$; in this range DLS results are insensitive to the fractal nature of the aggregates. The measured diffusion coefficient is due to center of mass diffusion. For the general case of polydispersed, flexible clusters $q R_{g} \gg 1$ and $\Gamma_{c} \propto q^{3}$. This latter behavior has been observed in a variety of aggregating systems such as colloidal polystyrene particles and microemulsions [12-14].

Figure 2 shows the measured $\Gamma_{c} / q^{2}$ versus $q$ for the investigated solution after aggregation was completed. As can be observed, in the present system, the first cumulant retains a $q^{2}$ dependence even in the $q R_{g} \gg 1$

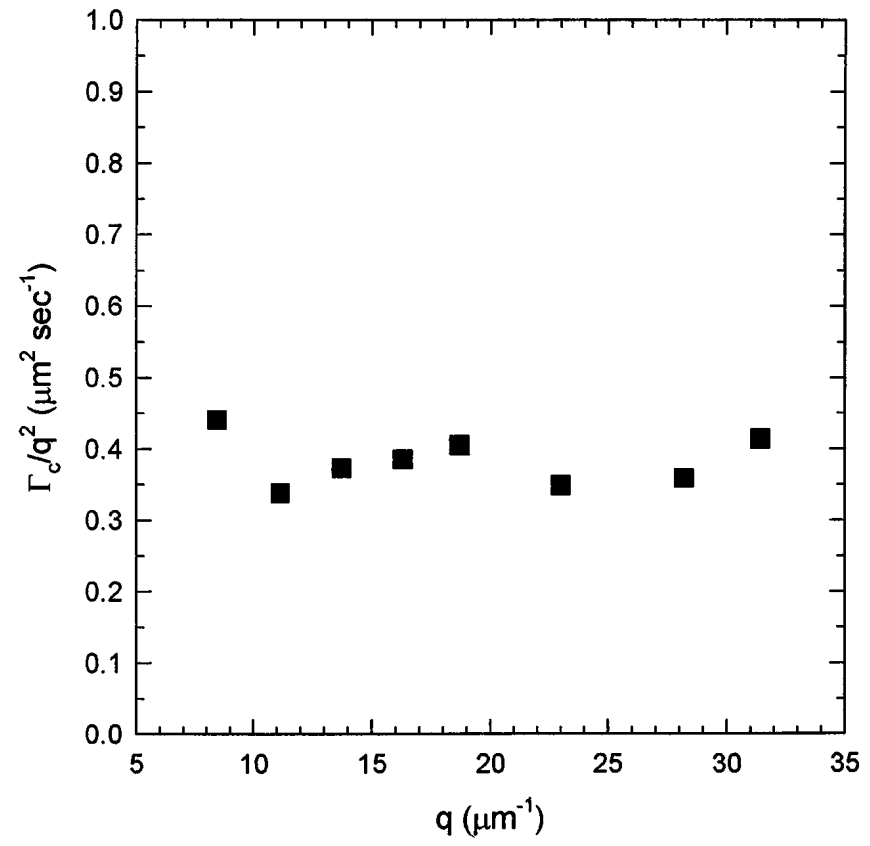

FIG. 2. Plot of $\Gamma_{c} / q^{2}$ vs $q$.

range. Such a $q^{2}$ dependence is very interesting as it reflects the structural properties observed in intensity data for dynamics measurements. A $q^{2}$ dependence in $\Gamma_{c}$ can provide information related to the properties of the cluster aggregate: their internal anisotropy, and intrinsic structural rigidity [15]. For the $q^{2}$ dependence to be observed, the aggregate must have a characteristic mass size $M_{c}$, which in turn sets an effective length in the size distribution [16]. The aggregates thus have a high degree of internal rigidity and are nearly monodisperse. Considering the optical anisotropies of a monodisperse cluster, it has been shown [15] that Eq. (5) can written as

$$
\Gamma_{c}(q)=D q^{2}\left[1+\frac{\alpha^{2}}{4 \beta^{2}}\right],
$$

where $\beta=R_{H} / R_{g}$ is the ratio between the hydrodynamic radius and the radius of gyration of the cluster and $\alpha$ is a quantity directly related to the anisotropy of the aggregate. From Eq. (6) we can consider $\Gamma_{c} / q^{2}$ as an effective diffusion coefficient $D_{\text {eff }}$. When $q R_{g} \ll 1$ it has a value of $D$ (the translational diffusion coefficient) and rises up to a higher limit for $q R_{g} \gg 1$. The value of this higher limit depends on the ratio of $R_{H}$ to $R_{g}$ for the cluster. It is well established $[16,17]$ that the value of the ratio depends on the cutoff function [Eq. (2)]. A step function yields $R_{H} / R_{g} \simeq 1.03$, whereas an exponential cutoff gives $R_{H} / R_{g} \simeq 0.62$.

It is important to note that we can assume that the diffusion coefficient $D$ is described by a single hydrodynamic radius $R_{H}$ using the Stokes relation $D=$ $k_{B} T / 6 \pi \eta R_{H}$. This approximation is suitable as long 
as the overall anisotropy of the cluster is not too large, a condition met experimentally $[18,19]$. In particular, from theoretical calculations for colloidal systems, $\alpha$ is expected to be 1.5 , a value confirmed from many experimental measurements [15]. The radius of gyration $R_{g}$ of a cluster can be obtained from the $\xi$ and $d_{f}$ values obtained from SALS and ELS data. For a fractal structure [19],

$$
R_{g}^{2}=\frac{d_{f}}{d_{f}+2} R^{2},
$$

where $R$ is the distance at which the cluster density becomes zero, i.e., $R \cong \xi$. By using the above equation, a value of about $2.1 \mu \mathrm{m}$ is obtained for the radius of gyration. With this value for $R_{g}$ and $\alpha=1.5$ and using Eq. (6) the ratio $\beta$ between the characteristic radii from the measured mean decay rate can be evaluated. The obtained value is $\beta=0.65 \pm 0.05$ which indicates an exponential cutoff in the density correlation function [Eq. (2)] and is in complete agreement with the findings of the elastic scattering data.

In summary, we have obtained by light scattering measurements information that the $t-\mathrm{H}_{2} \mathrm{P}_{\mathrm{agg}}$ porphyrin gives rise to large fractal clusters in aqueous solution. The aggregation phenomenon follows simple DLA kinetics giving rise to rigid monodisperse clusters. This rigidity of the clusters arises from the relatively strong dispersive forces acting in a system of porphyrin molecules. Porphyrins are compounds possessing an extended $\pi$ system in which so-called $\pi-\pi$ or stacking interactions take place. Such interactions lead to two different spatial arrangements [20]: an offset $\pi$ stacked, in which two molecules overlap each other with a lateral offset, and a T-shaped geometry, in which one molecule is rotated by $90^{\circ}$ with respect to the other one. This interaction and the strength of the electrostatic forces acting among prophyrin molecules are proposed to be responsible for the pronounced rigidity of the resulting aggregates. These conditions lead to the $q^{2}$ dependence observed in the first cumulant of the correlation function and to the structural anisotropy, which lead to the agreement between the characteristic radii measured by both elastic and quasielastic light scattering.

The authors are indebted to Professor D. A. Weitz for helpful suggestions. This work was supported by CNR through the "Progetto Finalizzato Technologie
Elettroottiche" and by NATO Grant No. SRG 950676. The research of F. M. is supported by the INFM.

[1] See, e.g., Structure and Dynamics of Strongly Interacting Colloids and Supramolecular Aggregates in Solution, edited by S.H. Chen, J.S. Huang, and P. Tartaglia (Luwer, Dordrecht, 1992); Scaling Concepts and Complex Fluids, F. Mallamace (Compositori, Bologna, 1995).

[2] See, e.g., On Growth and Form, edited by H. E. Stanley and N. Ostrowsky (Martinus Nijhoff Publishers, Dordercht, 1986).

[3] B. Mandlbrot, Fractals, Form and Dimensions (Freeman, San Francisco, 1977).

[4] R. F. Pasternak and E. J. Gibbs, ACS Symp. Ser. 402, 59 (1989); J. Inorg. Organomet. Polym. 3, 77 (1993).

[5] R. F. Pasternack, A. Giannetto, P. Pagano, and E. J. Gibbs, J. Am. Chem. Soc. 113, 7799 (1991).

[6] R.F. Pasternack, C. Bustamante, P. J. Collings, A. Giannetto, and E. J. Gibbs, J. Am. Chem. Soc. 115, 5393 (1993).

[7] F. Mallamace, N. Micali, S. Trusso, and S.H. Chen, Phys. Rev. E 51, 5818 (1995); S. H. Chen, D. Lombardo, F. Mallamace, N. Micali, S. Trusso, and C. Vasi, Prog. Colloid Polym. Sci 93, 311 (1993).

[8] B. J. Berne and R. Pecora, Dynamic Light Scattering (Wiley, New York, 1976).

[9] J. E. Martin and B.J. Akerson, Phys. Rev. A 31, 1180 (1985).

[10] S. H. Chen and J. Texeira, Phys. Rev. Lett. 57, 2583 (1986).

[11] S. K. Sinha, T. Freltoft, and J. Kjems, in Kinetic of Aggregation and Gelation, edited by F. Family and D. P. Landau (Elsevier, Amsterdam, 1984).

[12] J.E. Martin and D. W. Shaefer, Phys. Rev. Lett. 57, 2583 (1986); S. H. Chen, J. Rouch, P. Tartaglia, and F. Sciortino, Il Nuovo Cimento 16 D, 1419 (1994).

[13] F. Mallamace and N. Micali, La Rivista del Nuovo Cimento 15, 1-105 (1992).

[14] D. W. Shaefer and C. C. Han, in Dynamic Light Scattering, edited by R. Pecora (Plenum, New York, 1985).

[15] H. M. Lindsay, R. Klein, D. A. Weitz, M. Y. Lin, and P. Meakin, Phys. Rev. A 39, 3112 (1989).

[16] D. A. Weitz, J.S. Huang, M. Y. Lin, and J. Sung, Phys. Rev. Lett. 57, 2583 (1986).

[17] P. Wiltzius, Phys. Rev. Lett. 58, 710 (1987).

[18] W. van Saarloos, Physica (Amsterdam) 147A, 280 (1987).

[19] P. B. Warren, Il Nuovo Cimento 16 D, 1231 (1994).

[20] C. A. Hunter and M. K. J. Sanders, J. Am. Chem. Soc. 112, 5525 (1990). 